VOL. 18 (1978), 407-420.

\title{
Further results on an integral representation of functions of generalised variation
}

\section{A.M. Russell}

In this paper we present further properties of the $k$ th variation of a function, and obtain an integral representation for a function having bounded $k$ th variation and an absolutely continuous $(k-1)$ th derivative. The absolute continuity requirement replaces a previous stronger condition that required the $k$ th derivative of a function to be continuous except on a set of Lebesgue measure zero.

\section{Introduction}

It is a well known result that if $f$ is an absolutely continuous function on $[a, b]$, then $f$ is of bounded variation, and its variation is given by

$$
V_{1}(f ; a, b)=\int_{a}^{b}\left|f^{\prime}(t)\right| d t .
$$

In [3] the author extended this result to functions which have bounded $k$ th variation and which have the additional restriction that the $k$ th derivative is continuous except on a set of Lebesgue measure zero. In this paper we weaken the additional restriction by showing that the $k$ th total variation of a function $f$ can be written in the form

Received 6 March 1978. The author thanks Dr C.J.F. Upton for stimulating discussions relating to the contents of this paper. 


$$
(k-1) ! V_{k}(f ; a, b)=\int_{a}^{b}\left|f^{(k)}(t)\right| d t
$$

when $f^{(k-1)}$ is absolutely continuous on $[a, b]$.

In order to arrive at the more general result just outlined it was found expedient to work with two definitions of bounded $k$ th variation, one defined with quite arbitrary subdivisions $a=x_{0}, x_{1}, \ldots, x_{n}=b$ of $[a, b]$, and the other using subdivisions in which all subintervals. $\left[x_{i-1}, x_{i}\right]$ are of equal length. We show first that provided continuous functions are used, we obtain the same class of functions irrespective of which subdivisions are used.

\section{Notation and preliminaries}

DEFINITION 1. We shall say that a set of points $x_{0}, x_{1}, \ldots, x_{n}$ is a $\pi$-subdivision of $[a, b]$ when $a \leq x_{0}<x_{1}<\ldots<x_{n}=b$.

Before introducing the two definitions of bounded $k$ th variation, we need the definition and some properties of $k$ th divided differences, and for this purpose we refer the reader to [2].

DEFINITION 2. The total $k$ th variation of a function $f$ on $[a, b]$ is defined by

$$
V_{k}(f ; a, b)=\sup _{\pi} \sum_{i=0}^{n-k}\left(x_{i+k}-x_{i}\right)\left|Q_{k}\left(f ; x_{i}, \ldots, x_{i+k}\right)\right| .
$$

If $V_{k}(f ; a, b)<\infty$, we say that $f$ is of bounded $k$ th variation on $[a, b]$, and write $f \in B V_{k}[a, b]$. The sumations over which the supremum is taken are called approximating sums for $V_{k}(f ; a, b)$.

We now concern ourselves with subdivisions of $[a, b]$ in which all sub-intervals are of equal length. More formally, if $h>0$, then we will denote by $\pi_{h}$ a subdivision $x_{0}, x_{1}, \ldots, x_{n}$ of $[a, b]$ such that $a=x_{0}<x_{1}<\ldots<x_{n} \leq b$, where $x_{i}-x_{i-1}=h, i=1,2, \ldots, n$, and $0 \leq b-x_{n} \leq h$. In order to introduce the second definition of bounded $k$ th 
variation we make use of the difference operator $\Delta_{h}^{k}$ defined by

$$
\Delta_{h}^{I} f(x)=f(x+h)-f(x),
$$

and

$$
\Delta_{h}^{k} f(x)=\Delta_{h}^{1}\left(\Delta_{h}^{k-1} f(x)\right) .
$$

DEFINITION 3. If $f$ is continuous on $[a, b]$, then we define total $k$ th variation of $f$ on $[a, b]$ (restricted form) by

$$
\bar{V}_{k}(f ; a, b)=\sup _{\pi_{h}} \sum_{i=0}^{n-k}\left|\frac{\Delta_{h}^{k} f\left(x_{i}\right)}{h^{k-1}}\right| \text {. }
$$

If $\bar{V}_{k}(f ; a, b)<\infty$ we say that $f$ is of restricted bounded $k$ th variation on $[a, b]$, and write $f \in \overline{B V}_{k}[a, b]$.

If we denote, for brevity, $C[a, b]$ by $C, B V_{k}[a, b]$ by $B V_{k}$, and $\overline{B V}_{k}[a, b]$ by $\overline{B V}_{k}$, then we show subsequently that

$$
C \cap B V_{k}=\overline{B V}_{k} \text {. }
$$

We point out at this stage that the restriction to continuous functions is not nearly as severe as it first may appear, because functions belonging to $B V_{k}[a, b]$ when $k \geq 2$ are automatically continuous. (See Theorem 4 of [2].)

Our final definition deals with synchronized sets of points.

DEFINITION 4 . Let $x_{0}, x_{1}, \ldots, x_{n}$ and $y_{0}, y_{1}, \ldots, y_{n}$ be two sets of points belonging to $[a, b]$ such that $x_{0}<x_{1}<\ldots<x_{n}$ and $y_{0}<y_{1}<\ldots<y_{n} \cdot$ If

$$
y_{i}=x_{i+1}, i=0,1, \ldots, n-1
$$

or

$$
x_{i}=y_{i+1}, i=0,1, \ldots, n-1,
$$

we say that the two sets of points are synchronized; otherwise, we say 
that the two sets of points are not synchronized.

The following theorem will be a useful result. Since it is well known, and appears in the literature, for example, in $\$ 18$ of [1], a proof will not be given.

THEOREM 1. Let $F$ be absolutely continuous on $[a, b]$, written in the form $F(x)=\int_{a}^{x} f(t) d t, a \leq x \leq b$. Then $F$ is of bounded variation on $[a, b]$, and

$$
V_{1}(E ; a, b)=\int_{a}^{b}|f(t)| d t
$$

We now direct our attention to establishing the result

$$
C \cap B V_{k}=\overline{B V}_{k}, k \geq 1 \text {. }
$$

LEMMA 1. Let $I_{1}, I_{2}, \ldots, I_{n}$ be a set of $n$ adjoining closed intervals on the real line having lengths $p_{1} / q_{1}, p_{2} / q_{2}, \ldots, p_{n} / q_{n}$ respectively, where $p_{1}, p_{2}, \ldots, p_{n}, q_{1}, q_{2}, \ldots, q_{n}$ are positive integers. Then it is possible to subdivide the intervals $I_{1}, I_{2}, \ldots, I_{n}$ into sub-intervals of equal length.

The proof is easy and will be omitted.

LEMMA 2. If $k \geq 1$, then $C \cap B V_{k} \subset \overline{B V}_{k}$, using abbreviated notation.

Proof. This is easy and will not be included.

LEMMA 3. If $k \geq 1$, then $C \cap B V_{k} \supset \overrightarrow{B V}_{k}$.

Proof. Let us suppose that $f$ is continuous, belongs to $\overline{B V}_{k}[a, b]$, but $f \notin B V_{k}[a, b]$. Then for an arbitrarily large number $K$, and an arbitrarily small positive number $\varepsilon$, there exists a subdivision $\pi_{1}\left(x_{0}, x_{1}, \ldots, x_{n}\right)$ of $[a, b]$ such that

$$
S_{\pi_{1}} \equiv \sum_{i=0}^{n-k}\left(x_{i+k}{ }^{-x_{i}}\right)\left|Q_{k}\left(f ; x_{i}, \ldots, x_{i+k}\right)\right|>K+\varepsilon .
$$


If not all the lengths $\left(x_{i+1}{ }^{-x_{i}}\right), i=0,1, \ldots, n-1$ are rational, then because $f$ is continuous we can obtain a subdivision $\pi_{2}\left(y_{0}, y_{1}, \ldots, y_{n}\right)$ of $[a, b]$ in which all the lengths $\left(y_{i+1}-y_{i}\right), i=0,1, \ldots, n-1$ are rational, and such that $\left|S_{\pi_{1}}-S_{\pi_{2}}\right|<\varepsilon, S_{\pi_{2}}$ being the approximating sum of $V_{k}(f ; a, b)$ corresponding to the $\pi_{2}$ subdivision. Consequently,

$$
\begin{aligned}
S_{\pi_{2}} & \geq S_{\pi_{1}}-\left|S_{\pi_{1}}-S_{\pi_{2}}\right| \\
& >K .
\end{aligned}
$$

In the $\pi_{2}$ subdivision, all sub-intervals have rational length, so we can apply Lemma 1 to obtain a $\pi_{h}$ subdivision of $[a, b]$ in which each subinterval has length $h$. If $S_{\pi_{h}}$ is the corresponding approximating sum for $\bar{V}_{k}(f ; a, b)$, then it follows from Theorem 3 of [2] that

$$
\frac{1}{(k-1) !} S_{\pi_{h}} \geq S_{\pi_{2}}>K
$$

since for any $\pi_{h}$ subdivision, and each $i=0,1, \ldots, n-k$,

$$
\frac{\Delta_{h}^{k} f\left(x_{i}\right)}{h^{k-1}}=(k-1) !\left(x_{i+k}-x_{i}\right) Q_{k}\left(f ; x_{i}, \ldots, x_{i+k}\right) \text {. }
$$

Thus $S_{\pi_{h}}>(k-1) ! K$, and this is a contradiction to the assumption that $f \in \overline{B V}_{k}[a, b]$. Hence $f \in \overline{B V}_{k}[a, b]$, and so $\overline{B V}_{k} \subset C \cap B V_{k}$.

THEOREM 2. If $k \geq 1$, then $C \cap B V_{k}=\overline{B V}_{k}$; and if $f$ is a continuous function on $[a, b]$, then

$$
\bar{V}_{k}(f ; a, b)=(k-1) ! V_{k}(f ; a, b), k \geq 1 .
$$

Proof. The first part follows from Lemmas 2 and 3 . For the second part we first observe that

$$
\bar{V}_{k}(f ; a, b) \leq(k-1) ! V_{k}(f ; a, b) .
$$

Let $\varepsilon>0$ be arbitrary. Then there exists a $\pi_{1}$ subdivision of $[a, b]$ 
and the corresponding approximating sum $S_{\pi_{1}}$ to $V_{k}(f ; a, b)$ such that

$$
S_{\pi_{1}}>V_{k}(f ; a, b)-\frac{\varepsilon}{2(k-1) !}
$$

If not all the sub-intervals of $\pi_{1}$ have rational lengths, then we can proceed as in Lema 3 to obtain a $\pi_{h}$ subdivision of $[a, b]$ in which all sub-intervals are of equal length $h$. Then, if $S_{\pi_{h}}$ is the corresponding approximating sum to $\bar{V}_{k}(f ; a, b)$, we can show that

$$
\begin{aligned}
\frac{1}{(k-1) !} S_{\pi_{h}} & \geq S_{\pi_{1}}-\frac{\varepsilon}{2(k-1) !} \\
& >V_{k}(f ; a, b)-\frac{\varepsilon}{(k-1) !} .
\end{aligned}
$$

Consequently,

$$
\begin{aligned}
\bar{\nabla}_{k}(f ; a, b) & >S_{\pi_{h}} \\
& >(k-1) ! V_{k}(f ; a, b)-\varepsilon,
\end{aligned}
$$

from which it follows that $\bar{V}_{k}(f ; a, b) \geq(k-1) ! V_{k}(f ; a, b)$. This inequality together with (2) gives (1).

We now proceed towards an application of the result, $C \cap B V_{k}=\overrightarrow{B V}_{k}$.

\section{Main results}

Let the set of points $a=x_{0}, x_{1}, \ldots, x_{n-1}, x_{n}=b$ be a $\pi$ subdivision of $[a, b]$, and let $t$ be a real number such that $0 \leq t \leq 1$. We shall have need to consider the two related sets of points

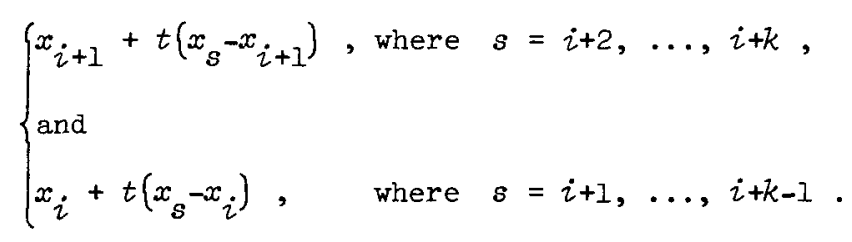

In relation to the sets of points (3) we shall consider the sum 
(4) $\sum_{i=0}^{n-k} \mid Q_{k-2}\left(f ; x_{i+1}+t\left(x-x_{i+1}\right) ; x_{i+2}, \ldots, x_{i+k}\right)-$

$$
-Q_{k-2}\left(f ; x_{i}+t\left(x-x_{i}\right) ; x_{i+1}, \ldots, x_{i+k-1}\right) \mid \text {. }
$$

Normally, the sum (4) would be an approximating sum for $V_{k-1}(f ; a, b)$, but since the two sets of points (3) are not synchronized subdivisions, further investigation is required to determine the relationship between (4) and $V_{k-1}(f ; a, b)$. In view of Theorem 2, we simplify our procedure by considering $\pi_{h}$ subdivisions in which each sub-interval $\left[x_{i-1}, x_{i}\right]$ is of length $h$. When $k \geq 2$ and $f \in B V_{k}[a, b], f$ is continuous, and so by Theorem 2, there is no loss of generality in considering $\pi_{h}$ subdivisions. Thus we can write (3) in the more convenient form

$$
x_{i+1}+t h, x_{i+1}+2 t h, \ldots, x_{i+1}+(k-1) t h \text {, }
$$

and

$$
x_{i}+\text { th }, x_{i}+2 t h, \ldots, x_{i}+(k-1) t h \text {. }
$$

The relative distribution of these two sets of points depends upon the value of $t$, so we now discuss various cases, starting with the simplest.

The case $t=0$. This is trivial as each divided difference in (4) is zero when $t=0$.

The case $0<t \leq \frac{1}{k-2}$. This gives rise to the distribution $x_{i}+t h<x_{i}+2 t h<\ldots<x_{i}+(k-1)$ th

$$
\leq x_{i+1}+t h<x_{i+1}+2 t h<\ldots<x_{i+1}+(k-1) t h .
$$

That $(4)$ is again dominated by $V_{k-1}(f ; a, b)$ follows readily. The cases $\frac{1}{p}<t \leq \frac{1}{p-1}, p=k-3, \ldots, 2$ are similar in character, with the "overlap" of the two sets "increasing" as $p$ decreases. We discuss in some detail the situation when $p=2$.

The case $\frac{1}{2}<t \leq 1$. First of all if $t=1$, (4) is clearly dominated by $v_{k-1}(f ; a, b)$. Hence we suppose that $\frac{1}{2}<t<1$, and present the following: 
THEOREM 3. If $\frac{1}{2}<t<1$, then

(5) $\begin{aligned} \sum_{i=0}^{n-k} \mid Q_{k-2} & \left(f ; x_{i+1}+t\left(x-x_{i+1}\right) ; x_{i+2}, \ldots, x_{i+k}\right)- \\ & -Q_{k-2}\left(f ; x_{i}+t\left(x-x_{i}\right) ; x_{i+1}, \ldots, x_{i+k-1}\right) \mid \leq v_{k-1}(f ; a, b) .\end{aligned}$

Proof. Suppose that $t$ is irrational, so that points of different sub-divisions do not coincide.

Let $n-k=1$, so that we consider the three sets of points

$$
x_{i}+t h, x_{i}+2 t h, \ldots, x_{i}+(k-1) t h, i=0,1,2 \text {. }
$$

The sets. of points corresponding to $i=0$ and $i=1$ are distributed relative to one another as follows:

$x_{0}+t h<x_{0}+2 t h<x_{1}+t h<x_{0}+3 t h<x_{1}+2 t h<\ldots$

$$
\ldots<x_{1}+(k-3) \text { th }<x_{0}+(k-1) \text { th }<x_{1}+(k-2) \text { th }<x_{1}+(k-1) \text { th. }
$$

In other words, after the first two points $x_{0}+t h$ and $x_{0}+2 t h$, the points alternate until $x_{0}+(k-1)$ th, and this is finally followed by $x_{1}+(k-2)$ th and $x_{1}+(k-1)$ th. However, when the third set of points is added some ambiguity occurs because $x_{2}+t h$, definitely greater than $x_{1}+2 t h$, may be either greater than or less than $x_{0}+4 t h$, depending upon the value of $t$ in $\left(\frac{1}{2}, 1\right)$. To be definite, let us assume that $x_{1}+2 t h<x_{2}+t h<x_{0}+4 t h$, and proceed. An analysis similar to the following will apply if we assume $x_{2}+t h>x_{0}+4 t h$. Accordingly, relabel the set of $(3 k-3)$ points $y_{1}, y_{2}, \ldots, y_{3 k-3}$, where $\begin{aligned} y_{1}=x_{0}+t h, y_{2} & =x_{0}+2 t h, y_{3}=x_{1}+t h, y_{4}=x_{0}+3 t h, \\ y_{5} & =x_{1}+2 t h, y_{6}=x_{2}+t h, y_{7}=x_{0}+4 t h, \ldots, y_{3 k-3}=x_{2}+(k-1) t h .\end{aligned}$ Consequently, using Theorem 1, Corollary of [2], and writing $Q_{k-2}\left(y_{i}, \ldots, y_{i+k-2}\right)$ instead of $Q_{k-2}\left(f ; y_{i}, \ldots, y_{i+k-2}\right)$, we obtain 


$$
\begin{aligned}
& \sum_{i=0}^{n-k} \mid Q_{k-2}\left(f ; x_{i}+t\left(x-x_{i}\right) ; x_{i+1}, \ldots, x_{i+k-1}\right)- \\
& \quad-Q_{k-2}\left(f ; x_{i+1}+t\left(x_{-x_{i+1}}\right) ; x_{i+2}, \ldots, x_{i+k}\right) \mid \\
& =\mid \beta_{1} Q_{k-2}\left(y_{1}, \ldots, y_{k-1}\right)+\beta_{2} Q_{k-2}\left(y_{2}, \ldots, y_{k}\right)+\ldots \\
& \ldots+\beta_{2 k-5} Q_{k-2}\left(y_{2 k-5}, \ldots, y_{3 k-7}\right)-\alpha_{3} Q_{k-2}\left(y_{3}, \ldots, y_{k+1}\right)- \\
& -\alpha_{4} Q_{k-2}\left(y_{4}, \ldots, y_{k+2}\right)-\ldots-\alpha_{2 k-3} Q_{k-2}\left(y_{2 k-3}, \ldots, y_{3 k-5}\right) \mid+ \\
& +\mid \alpha_{3} Q_{k-2}\left(y_{3}, \ldots, y_{k+1}\right)+\alpha_{4} Q_{k-2}\left(y_{4}, \ldots, y_{k+2}\right)+ \\
& \ldots+\alpha_{2 k-3} Q_{k-2}\left(y_{2 k-3}, \ldots, y_{3 k-5}\right)-\gamma_{5} Q_{k-2}\left(y_{5}, \ldots, y_{k+3}\right)- \\
& -\gamma_{6} Q_{k-2}\left(y_{6}, \ldots, y_{k+4}\right)-\ldots, \gamma_{2 k-1} Q_{k-2}\left(y_{2 k-1}, \ldots, y_{3 k-3}\right) \mid,
\end{aligned}
$$

where the $\alpha^{\prime} s, B^{\prime} s$, and $\gamma^{\prime} \bar{s}$ are all non-negative, and $\beta_{1}+\beta_{2}+\ldots+\beta_{2 k-5}=\alpha_{3}+\alpha_{4}+\ldots+\alpha_{2 k-3}=\gamma_{5}+\gamma_{6}+\ldots+\gamma_{2 k-1}=1$.

After some re-arrangement, the sumation can be shown to equal

$$
\begin{aligned}
\mid \beta_{1}\left\{Q \left(y_{1}, \ldots,\right.\right. & \left.\left.y_{k-1}\right)-Q\left(y_{2}, \ldots, y_{k}\right)\right\}+ \\
& +\left(\beta_{1}+\beta_{2}\right)\left\{Q\left(y_{2}, \ldots, y_{k}\right)-Q\left(y_{3}, \ldots, y_{k+1}\right)\right\} \\
& +\left(\beta_{1}+\beta_{2}+\beta_{3}-\alpha_{3}\right)\left\{Q\left(y_{3}, \ldots, y_{k+1}\right)-Q\left(y_{4}, \ldots, y_{k+2}\right)\right\}+ \\
& \ldots+\left(\beta_{1}+\beta_{2}+\beta_{3}+\ldots+\beta_{2 k-5}-\alpha_{3}-\alpha_{4}-\ldots-\alpha_{2 k-5}\right) \times \\
& \left\{Q\left(y_{2 k-5}, \ldots, y_{3 k-7}\right)-Q\left(y_{2 k-4}, \ldots, y_{3 k-6}\right)\right\}+ \\
& +\left(\beta_{1}+\ldots+\beta_{2 k-5}-\alpha_{3}-\alpha_{4}-\ldots-\alpha_{2 k-4}\right) \times \\
& \left\{Q\left(y_{2 k-4}, \ldots, y_{3 k-6}\right)-Q\left(y_{2 k-3}, \ldots, y_{3 k-5}\right)\right\} \mid \\
& +\mid \alpha_{3}\left\{Q\left(y_{3}, \ldots, y_{k+1}\right)-Q\left(y_{4}, \ldots, y_{k+2}\right)\right\}+ \\
& +\left(\alpha_{3}+\alpha_{4}\right)\left\{Q\left(y_{4}, \ldots, y_{k+2}\right)-Q\left(y_{5}, \ldots, y_{k+3}\right)\right\}+ \\
& +\left(\alpha_{3}+\alpha_{4}+\alpha_{5}-\gamma_{5}\right)\left\{Q\left(y_{5}, \ldots, y_{k+3}\right)-Q\left(y_{6}, \ldots, y_{k+4}\right)\right\}+ \\
& \ldots+\left(\alpha_{3}+\alpha_{4}+\ldots+\alpha_{2 k-3}-\gamma_{5}-\gamma_{6}-\ldots-\gamma_{2 k-3}\right\} \times \\
& \left\{Q\left(y_{2 k-3}, \ldots, y_{3 k-5}\right)-Q\left(y_{2 k-2}, \ldots, y_{3 k-4}\right)\right\}+ \\
& +\left(\alpha_{3}+\alpha_{4}+\ldots+\alpha_{2 k-3}-\gamma_{5}-\gamma_{6}-\ldots-\gamma_{2 k-2}\right\} \times \\
& \left\{Q\left(y_{2 k-2}, \ldots, y_{3 k-4}\right)-Q\left(y_{2 k-1}, \ldots, y_{3 k-3}\right)\right\} \mid \\
\leq \beta_{1} \mid Q\left(y_{1}\right. & \left.\ldots, y_{k-1}\right)-Q\left(y_{2}, \ldots, y_{k}\right) \mid+\left(\beta_{1}+\beta_{2}\right) \times
\end{aligned}
$$




$$
\begin{aligned}
& \left|Q\left(y_{2}, \ldots, y_{k}\right)-Q\left(y_{3}, \ldots, y_{k+1}\right)\right|+\left(\beta_{1}+\beta_{2}+\beta_{3}-\alpha_{3}+\alpha_{3}\right) \times \\
& \left|Q\left(y_{3}, \ldots, y_{k+1}\right)-Q\left(y_{4}, \ldots, y_{k+2}\right)\right|+\left(\beta_{1}+\beta_{2}+\beta_{3}+\beta_{4}-\alpha_{3}-\alpha_{4}+\alpha_{3}+\alpha_{4}\right) \times \\
& \left|Q\left(y_{4}, \ldots, y_{k+2}\right)-Q\left(y_{5}, \ldots, y_{k+3}\right)\right|+\ldots+ \\
& +\left(1-\alpha_{3}-\alpha_{4}-\ldots-\alpha_{2 k-4}+\alpha_{3}+\alpha_{4}+\ldots+\alpha_{2 k-4}-\gamma_{5}-\gamma_{6}-\gamma_{2 k-4}\right) \times \\
& \left|Q\left(y_{2 k-4}, \ldots, y_{3 k-6}\right)-Q\left(y_{2 k-3}, \ldots, y_{3 k-5}\right)\right|+ \\
& +\left(1-\gamma_{5}-\gamma_{6}-\ldots-\gamma_{2 k-3}\right)\left|Q\left(y_{2 k-3}, \ldots, y_{3 k-5}\right)-Q\left(y_{2 k-2}, \ldots, y_{3 k-4}\right)\right| \\
& +\left(1-\gamma_{5}-\gamma_{6}-\ldots-\gamma_{2 k-2}\right)\left|Q\left(y_{2 k-2}, \ldots, y_{3 k-4}\right)-Q\left(y_{2 k-1}, \ldots, y_{3 k-3}\right)\right| \\
& \leq \sum_{i=0}^{2 k-2}\left|Q\left(y_{i}, \ldots, y_{i+k-2}\right)-Q\left(y_{i+1}, \ldots, y_{i+k-1}\right)\right| \leq V_{k-1}(f ; a, b) .
\end{aligned}
$$

A similar, but longer, analysis applies for higher values of $n-k$.

Finally, let $t$ be a rational number. Then, since $f$ is contimuous, sets of points $x_{i}+s t^{\prime} h$ and $x_{i+1}+s t^{\prime} h, s=1,2, \ldots, k-1$, where $t^{\prime}$ is irrational, exist such that the sums (4) corresponding to $t$ and $t^{\prime}$ differ by an arbitrarily small specified $\varepsilon$. Thus (5) is still valid, and we conclude the proof.

THEOREM 4. If $k \geq 3$, and $f \in B V_{k}[a, b]$, then $f^{\prime} \in B V_{k-1}[a, b]$ and

$$
v_{k-1}\left(f^{\prime} ; a, b\right) \leq(k-1) v_{k}(f ; a, b)
$$

Proof. That $f^{\prime} \in B V_{k-1}[a, b]$ follows from Theorem 12 of [2]. Now see Theorem 9 of [2], but observe that the " $k^{2 "}$ in the second last line of the proof of that theorem can be replaced by " $k$ ".

THEOREM 5. If $k \geq 3$, and $f \in B V_{k}[a, b]$, then $f^{\prime} \in B V_{k-1}[a, b]$ and

$$
V_{k-1}\left(f^{\prime} ; a, b\right) \geq(k-1) V_{k}(f ; a, b) .
$$

Proof of inequality. It follows from Theorem 11 of [2] that $f^{\prime}$ is continuous in $[a, b]$, so we can write

$$
f(x)=f(a)+\int_{a}^{x} f^{\prime}(t) d t .
$$


Hence, using a property of $k$ th divided differences,

(7)

$$
\begin{aligned}
& \left|Q_{k-1}\left(f ; x_{i+1}, \ldots, x_{i+k}\right)-Q_{k-1}\left(f ; x_{i}, \ldots, x_{i+k-1}\right)\right| \\
& =\left|Q_{k-2}\left(\frac{f(x)-f\left(x_{i+1}\right)}{x-x_{i+1}} ; x_{i+2}, \ldots, x_{i+k}\right)-Q_{k-2}\left\{\frac{f(x)-f\left(x_{i}\right)}{x_{-1}} ; x_{i+1}, \ldots, x_{i+k-1}\right)\right| \\
& =\mid Q_{k-2}\left(\int_{0}^{1} f^{\prime}\left(x_{i+1}+t\left(x_{i+1}\right)\right) d t ; x_{i+2}, \ldots, x_{i+k}\right\} \\
& =\mid \int_{0}^{1}\left\{Q_{k-2}\left(f^{\prime}\left(x_{i+1}+t\left(x-x_{i+1}\right)\right) ; x_{i+2}, \ldots, x_{i+k}\right)\right. \\
& \quad-Q_{k-2}\left(\int_{0}^{1} f^{\prime}\left(x_{i}+t\left(x_{-x_{i}}\right)\right) d t ; x_{i+1}, \ldots, x_{i+k-1}\right\} d t \mid \\
& =\left|\int_{0}^{1}\left\{Q_{k-2}\left(f^{\prime}(x) ; x_{i+1}+t\left(x_{i+2}-x_{i+1}\right), \ldots, x_{i+1}+t\left(x_{i+k}+t\left(x_{i+1}\right)\right) ; x_{i+1}, \ldots, x_{i+k-1}\right)\right\} d t\right| \\
& \left.\quad-Q_{k-2}\left(f^{\prime}(x) ; x_{i}+t\left(x_{i+1}-x_{i}\right), \ldots, x_{i}+t\left(x_{i+k-1}-x_{i}\right)\right)\right\} \mid t^{k-2} d t .
\end{aligned}
$$

Therefore, using Theorem 3, we obtain

$$
\begin{aligned}
& \sum_{i=0}^{n-k}\left|Q_{k-1}\left(f ; x_{i+1}, \ldots, x_{i+k}\right)-Q_{k-1}\left(f ; x_{i}, \ldots, x_{i+k=1}\right)\right| \\
& \leq V_{k-1}\left(f^{\prime} ; a, b\right) \int_{0}^{1} t^{k-2} d t=\frac{1}{k-1} V_{k-1}\left(f^{\prime} ; a, b\right) .
\end{aligned}
$$

We can now conclude that

$$
(k-1) V_{k}(f ; a, b) \leq V_{k-1}\left(f^{\prime} ; a, b\right),
$$

as required.

Combining (6) and (8) gives us

THEOREM 6. If $k \geq 3$, and $f \in B V_{k}[a, b]$, then $f^{\prime} \in B V_{k-1}[a, b]$, and

$$
V_{k-1}\left(f^{\prime} ; a, b\right)=(k-1) V_{k}(f ; a, b)
$$

We now treat the case $k=2$ separately, this case requiring the 
extra hypothesis that $f^{\prime}$ exists throughout $[a, b]$.

THEOREM 7. If $f \in B V_{2}[a, b]$ and $f^{\prime}$ exists in $[a, b]$, then $f^{\prime} \in B V[a, b]$ and

$$
V_{2}(f ; a, b)=V_{1}\left(f^{\prime} ; a, b\right) .
$$

Proof. It follows from Theorem 9 of [2] that

$$
V_{1}\left(f^{\prime} ; a, b\right) \leq V_{2}(f ; a, b) \text {. }
$$

To establish the reverse inequality, let $a=x_{0}, x_{1}, \ldots, x_{n}=b$ be any subdivision of $[a, b]$. Then

$$
\begin{aligned}
& \sum_{i=0}^{n-2}\left|Q_{1}\left(f ; x_{i+1}, x_{i+2}\right)-Q_{1}\left(f ; x_{i}, x_{i+1}\right)\right| \\
& \quad=\sum_{i=0}^{n-2}\left|f^{\prime}\left(\eta_{i+1}\right)-f^{\prime}\left(\eta_{i}\right)\right|, \text { where } x_{i}<\eta_{i}<x_{i+1}, i=0,1, \ldots, n-2, \\
& \quad \leq V_{1}\left(f^{\prime} ; a, b\right) .
\end{aligned}
$$

Therefore,

$$
V_{2}(f ; a, b) \leq V_{1}\left(f^{\prime} ; a, b\right) .
$$

From (10) and (1I) it is now clear that

$$
v_{2}(f ; a, b)=V_{1}\left(f^{\prime} ; a, b\right) .
$$

We are now in a position to offer more general versions of Theorems 3 and 4 of [3].

THEOREM 8. If $f \in B V_{k}[a, b], k \geq 3$, then

$$
(k-1) ! V_{k}(f ; a, b)=V_{2}\left(f^{(k-2)} ; a, b\right) \text {. }
$$

Furthermore, if $k \geq 2$, and $f^{(k-1)} \in B V_{1}[a, b]$, then

$$
(k-1) ! V_{k}(f ; a, b)=V_{1}\left(f^{(k-1)} ; a, b\right) .
$$

Proof. It follows from Theorem 12 of [2] that $f^{(p)} \in B V_{k-r}[a, b]$, $r=1,2, \ldots, k-2$. Successive applications of (9), and a final 
application of Theorem 7 , give the required results.

THEOREM 9. Let $f$ be a function such that $f^{(k-1)}$ is absolutely continuous on $[a, b]$. Then $f^{(k-s)} \in B V_{s}[a, b], s=1,2, \ldots, k$, and, in particular,

$$
(k-1) ! V_{k}(f ; a, x)=\int_{a}^{x}\left|f^{(k)}(t)\right| d t, \quad a \leq x \leq b .
$$

Proof. Since $f^{(k-1)}$ is absolutely continuous on $[a, b]$, it is also of bounded (first) variation on that interval. It follows from repeated applications of Lemma 3 of [4] that

$$
f^{(k-s)} \in B V_{s}[a, b], s=1,2, \ldots, k .
$$

Consequently, from the second part of the previous theorem, we conclude that

$$
(k-1) ! v_{k}(f ; a, b)=V_{1}\left(f^{(k-1)} ; a, b\right) \text {, }
$$

and

$$
V_{1}\left(f^{(k-1)} ; a, b\right)=\int_{a}^{b}\left|f^{(k)}(t)\right| d t
$$

using Theorem 1 .

REMARK. In view of (1), (13) can be written in the more elegant form

$$
\bar{v}_{k}(f ; a, x)=\int_{a}^{x}\left|f^{(k)}(t)\right| d t, \quad a \leq x \leq b .
$$

\section{References}

[1] Edwin Hewitt, Karl Stromberg, Real and abstract analysis. A moderm treatment of the theory of functions of a real variable (Springer-Verlag, Berlin, Heidelberg, New York, 1965. Second printing corrected: Springer-Verlag, Berlin, Heidelberg, New York, 1969. Third printing: Graduate Texts in Mathematics, 25. Springer-Verlag, New York, Heidelberg, Berlin, 1975). 
[2] A.M. Russell, "Functions of bounded kth variation", Proc. London Math. Soc. (3) 26 (1973), 547-563.

[3] A.M. Russell, "An integral representation for a generalised variation of a function", Bulz. Austral. Math. Soc. 11 (1974), 225-229.

[4] A.M. Russell, "Stieltjes-type integrals", J. Austral. Math. Soc. Ser. A 20 (1975), 431-448.

Department of Mathematics,

University of Melbourne,

Parkville,

victoria. 